\title{
Actively Q-Switched All-Fiber Laser with a Fiber Bragg Grating of Variable Reflectivity
}

\author{
Ding-Wei Huang \\ Department of Electrical Engineering and Graduate Institute of Electro-Optical Engineering, \\ National Taiwan University, Taipei, Taiwan, R.O.C. \\ Wen-Fung Liu \\ Department of Electrical Engineering, Chung Cheng Institute of Technology, Tahsi, Taoyuan, \\ Taiwan, R.O.C. \\ C. C. Yang \\ Department of Electrical Engineering and Graduate Institute of Electro-Optical Engineering, \\ National Taiwan University, 1, Roosevelt Road, Sec. 4, Taipei, Taiwan, R.O.C. \\ phone: 886-2-23657624 fax: 886-2-23652637 E-mail: ccy@cc.ee.ntu.edu.tw
}

Q-switching of erbium-doped fiber laser can provide simple and robust high-pulse-energy light sources for various applications, including eye-safe rarging, remote sensing, medicine, and others. Typically, external-cavity acousto-optical modulation was used for implementing Qswitching. However, the required external-cavity configuration represents a drawback, which is open for improvement. It is expected that an all-fiber Q-switched laser can increase the efficiency and its robustness. In this paper, we report a novel Q-switched fiber laser based on an acoustically modulated fiber Bragg grating reflector. We used a fiber Bragg grating as the end mirror of the laser cavity. This fiber grating was under acoustic perturbation for controlling its Bragg reflectivity. Therefore, the Q-factor of the laser cavity was switched high and low by the acoustic wave. Pulses of around $150 \mathrm{nsec}$ in width and $7.6 \mu \mathrm{J}$ in pulse energy were obtained. The laser slope efficiency was $13.1 \%$. This all-fiber Q-switched fiber laser can be quite compact and robust.

Figure 1 shows the experimental setup of the Q-switched fiber laser. Right to the $72-\mathrm{cm}$ long erbium-doped fiber, it was spliced to a fiber Bragg grating with the Bragg wavelength at $1550.5 \mathrm{~nm}$. Between the grating and erbium-doped fiber, the fiber was laterally glued onto the tip of a metal horn (made of aluminum), whose base was attached to a piezoelectric transducer (PZT), driven by a voltage source, for receiving mechanical vibration. This is the part of the setup for producing acoustic perturbation of the fiber grating. Left to the erbium-doped fiber, a WDM fiber coupler was connected for providing $980 \mathrm{~nm}$ pump power, which was provided by an argon-laser pumped Ti:sapphire laser. To the very left end, the cleaved fiber facet was used as the output coupler of the laser and provided about $4 \%$ reflectivity. The whole cavity length was $136 \mathrm{~cm}$. 
The fiber grating has a length of $1.5 \mathrm{~cm}$ and a Bragg reflection window of $0.5 \mathrm{~nm}$, centered at $1550.5 \mathrm{~nm}$. The peak reflectivity was about $95 \%$. To concentrate the incoming acoustic power near the core of the fiber, the cladding of the fiber grating section was etched with HF solution to reduce the cladding diameter down to $30 \mu \mathrm{m}$. The length of this minimum cladding diameter was $2 \mathrm{~cm}$ with the $1.5-\mathrm{cm}$ long grating located at the center. Including the two taper sections on both sides, the whole etched fiber length was about $5 \mathrm{~cm}$. The metal horn was $1 \mathrm{~cm}$ in base diameter and $1.5 \mathrm{~cm}$ in length. Its tip was glued to the fiber about $2.5 \mathrm{~cm}$ from the grating center. Its base was glued to a thin PZT of $1.3 \mathrm{~cm}$ in diameter and $1.8 \mathrm{~mm}$ in thickness. The PZT was connected to a voltage source for driving acoustic waves. The acoustic waves propagated with increasing intensity along the horn to its tip and translated the power to the fiber. With the acoustic excitation, the fiber vibrated transversely to create micro-bending. The micro-bending produced the optical coupling between the core mode and cladding modes of the fiber. The cladding-mode power decayed through radiation. The out-coupling of optical core-mode signal reduced the power for Bragg reflection of the grating. Therefore, Bragg reflectivity of the fiber grating decreased with the intensity of the acoustic wave or the applied voltage to the PZT. Figure 2 shows the variation of Bragg reflection spectrum with various applied voltage levels when the acoustic frequency is $713 \mathrm{kHz}$. One can see that as the peak-to peak (p-p) voltage increases to $68.8 \mathrm{~V}$, the Bragg reflectivity near $1550.5 \mathrm{~nm}$ is reduced to almost zero. This is the acoustic perturbation mechanism for Q-switching.

To see the response speed of the acoustic control of Bragg reflectivity, we applied a periodical square-wave modulation with various frequencies to the sinusoidal voltage signal at $713 \mathrm{kHz}$. Figure 3 shows the modulation response when the modulation frequency is $5 \mathrm{kHz}$. Part (b) of this figure shows the applied sinusoidal voltage under $5 \mathrm{kHz}$ modulation with $50 \%$ duty cycle. Part (a) shows the variation of Bragg reflectivity at $1550.5 \mathrm{~nm}$ of the fiber grating. One can see that switch-off of Bragg reflectivity is faster than switch-on as the acoustic wave is turned on and then off. The switching time depends on several parameters, including sound speed in fiber, the location of the horn tip along the fiber, the length of fiber grating, and the masses of the metal horn and PZT. The reflectivity starts to recover about $40 \mu \mathrm{sec}$ after the voltage is turned off. Before turn-on of acoustic wave of the next cycle, only $60 \mu \mathrm{sec}$ left for reflectivity recovery. Because of the slow recovery and fast decay of Bragg reflectivity, the reflectivity value fluctuates between 0.06 and 0.27 . Note that because the sampling rate of the used oscilloscope was not well set, the fast oscillation of the applied voltage in Fig. 3 does not represent the real acoustic frequency.

Figure 4 shows the laser output pulse profile and the applied voltage. Here, the Q-switching frequency was $5 \mathrm{kHz}$, the applied voltage was $72 \mathrm{~V}$ (p-p), and the acoustic frequency was 713 $\mathrm{kHz}$. The $980 \mathrm{~nm}$ pump power was $290 \mathrm{~mW}$. This figure shows that the pulse width is about 150 
nsec. The laser threshold is near $2 \mathrm{~mW}$ and the slope efficiency is about $13.1 \%$. Take the case of $290 \mathrm{~mW}$ pump power as an example, it corresponds to the average output power of $38 \mathrm{~mW}$ or pulse energy of $7.6 \mu \mathrm{J} /$ pulse. With the pulse width of $150 \mathrm{nsec}$, the peak power is $50.7 \mathrm{~W}$. It is believed that the pulse energy can be scaled up for at least several times.

(a)

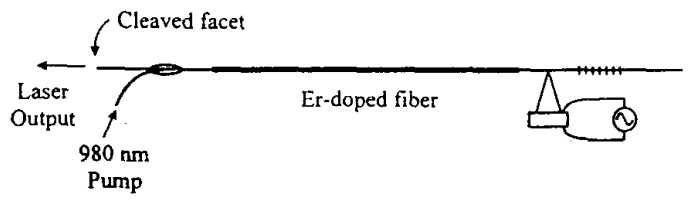

(b)

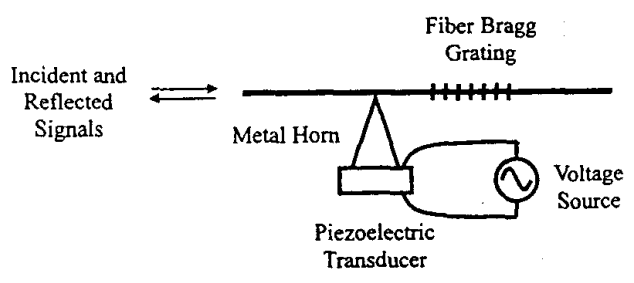

Fig. 1 Experimental setup of the Q-switched laser.

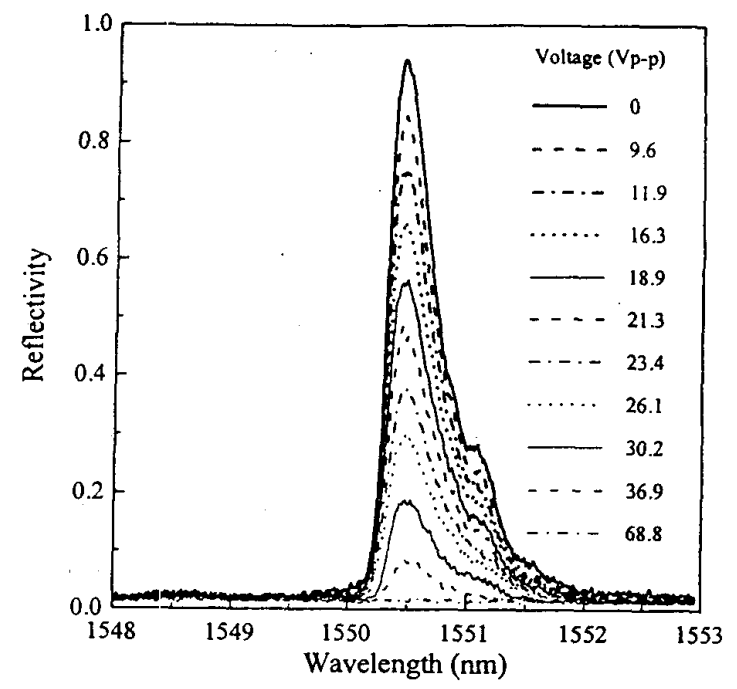

Fig. 2 Bragg reflection spectrum variation with various applied voltage levels.

(a)

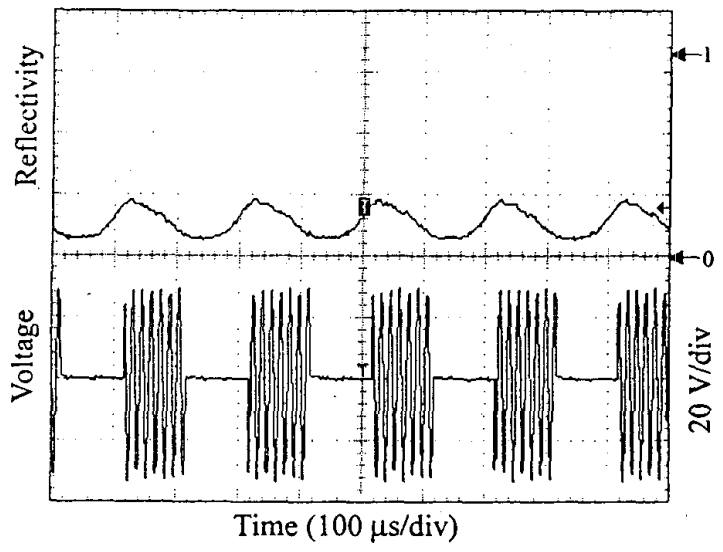

Fig. 3 (a) Bragg reflectivity response to a squarewave modulation of acoustic wave with modulation

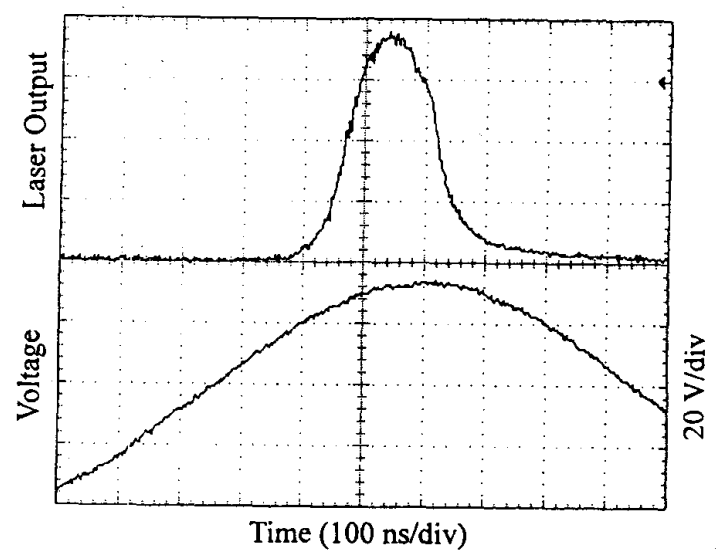

Fig. 4 Laser output pulse shape and the modulated applied voltage. 